\title{
Erratum to: Extremophiles: Applications in Nanotechnology
}

\section{Erratum to:}

S. Tiquia-Arashiro and D. Rodrigues,

Extremophiles: Applications in Nanotechnology, https://doi.org/10.1007/978-3-319-45215-9

In the original version of the book, the incorrect corresponding author name "Debora Frigit Rodrigues" has been changed as "Sonia Tiquia-Arashiro" for Chaps. 1-4 and "Debora Rodrigues" is the corresponding author for Chap. 5.

The updated online version of this book can be found at https://doi.org/10.1007/978-3-319-45215-9 